\title{
Least Squares Support Vector Machines for Direction of Arrival Estimation with Error Control and Validation
}

\author{
Judd A. Rohwer \\ Sandia National Laboratories \\ P.O. Box 5800 MS-0986 \\ Albuquerque, NM 87185-0986 \\ Email: jarohwe@sandia.gov
}

\author{
Chaouki T. Abdallah and Christos G. Christodoulou \\ Department of Electrical and Computer Engineering \\ MSC01 1100 \\ 1 University of New Mexico \\ Albuquerque, NM 87131-0001 \\ Email: [chaouki,christos]@eece.unm.edu
}

\begin{abstract}
This paper presents a multiclass, multilabel implementation of Least Squares Support Vector Machines (LS-SVM) for direction of arrival (DOA) estimation in a CDMA system. For any estimation or classification system the algorithm's capabilities and performance must be evaluated. Specifically, for classification algorithms a high confidence level must exist along with a technique to automatically tag misclassifications. The learning algorithm presented in this paper includes error control and validation steps for generating statistics on the multiclass evaluation path and the signal subspace dimension. The error statistics provide a confidence level of the classification accuracy.
\end{abstract}

\section{INTRODUCTION}

Machine learning research has largely been devoted to binary and multiclass problems relating to data mining, text categorization, and pattern/facial recognition. Recently, popular machine learning algorithms, including support vector machines (SVM), have successfully been applied to wireless communication problems, notably spread spectrum receiver design [1], and channel equalization [2].

Beamforming, tracking, and DOA estimation are current research topics with various technical approaches; three techniques based on signal subspace decomposition are ESPRIT, MUSIC, and Root-MUSIC [3]. In [4] neural networks have been successfully applied to the problem of DOA estimation and adaptive beamforming. New machine learning techniques, such as SVMs and boosting [5], perform exceptionally well in multiclass problems and new optimization techniques are published regularly. These new techniques have the potential to exceed the performance of the neural network algorithms relating to communication applications.

In this paper we present a multiclass SVM algorithm trained with projection vectors generated from the signal subspace eigenvectors and the respective covariance matrices. The output labels from the SVM system are the DOA estimates. The broad range of our research in LS-SVM based DOA estimation includes multilabel and multiclass classification, classification

\footnotetext{
${ }^{1}$ Sandia is a multiprogram laboratory operated by Sandia Corporation, a Lockheed Martin Company, for the United States Department of Energy under Contract DE-AC04-94AL85000.
}

accuracy, error control and validation, kernel selection, and estimation of signal subspace dimension.

\section{Least Squares Support Vector Machines}

SVMs were originally designed for binary classification problems. A variety of approaches are currently being developed to tackle the problem of applying SVMs to multiclass problems. Much like all machine learning algorithms SVMs find a classification function that separates data classes, with the largest margin, using a hyperplane. The data points near the optimal hyperplane are the "support vectors". SVMs are a nonparametric machine learning algorithm with the capability of controlling the capacity through the support vectors.

In a binary classification system the input sequence and a set of training labels are represented as $\left\{\mathbf{x}_{k}, y_{k}\right\}_{k=1}^{K}$, where $y_{k}=\{-1,1\}$ represents the classification "label" applied to the input vector $\mathbf{x}_{k}$. If the two classes are linearly separable in the input space then the hyperplane separating the classes is defined as $\mathbf{w}^{T} \mathbf{x}+b=0, \mathbf{w}$ is a weight vector perpendicular to the separating hyperplane, $b$ is a bias that shifts the hyperplane parallel to itself. If the input space is projected into a higher dimensional feature space then the hyperplane becomes $\mathbf{w}^{T} \boldsymbol{\Gamma}(\mathbf{x})+b=0$. The nonlinear function $\boldsymbol{\Gamma}(\cdot)$ maps the input space to the feature space.

Suykens, et.al., [6] introduced a least squares SVM (LSSVM) which is based on the SVM classifier,

$$
y(\mathbf{x})=\operatorname{sign}\left[\sum_{k=1}^{K} \alpha_{k} y_{k} \boldsymbol{\Gamma}\left(\mathbf{x}, \mathbf{x}_{k}\right)+b\right] .
$$

The LS-SVM classifier is generated from the optimization problem:

$$
\min _{\mathbf{w}, b, \phi} \mathcal{L}_{L S}(\mathbf{w}, \phi)=\frac{1}{2}\|\mathbf{w}\|^{2}+\frac{1}{2} \gamma \sum_{k=1}^{K} \phi_{k}^{2},
$$

$\gamma$ and $\phi_{k}$ are the regularization and error variables, respectively. The minimization in (2)includes the constraints

$$
y_{k}\left[\mathbf{w}^{T} \boldsymbol{\varphi}\left(\mathbf{x}_{k}\right)+b\right] \geq 1-\phi_{k}, \quad k=1, \ldots, K,
$$


Misclassifications, due to overlapping distributions, are accounted for with the error variables $\phi_{k}, \gamma$ is a regularization parameter that governs the complexity of the SVM, and the margin between the hyperplane and the data points in the feature space is maximize when $\mathbf{w}$ is minimized. The Lagrangian of equation (2) is defined as

$$
\begin{aligned}
\mathcal{Z}_{L S}(\mathbf{w}, b, \phi, \alpha)= & \mathcal{L}_{L S}(\mathbf{w}, b, \phi) \\
& -\sum_{k=1}^{K} \alpha_{k}\left\{y_{k}\left[\mathbf{w}^{T} \boldsymbol{\Gamma}\left(x_{k}\right)+b\right]-1+\phi_{k}\right\}
\end{aligned}
$$

where $\alpha_{k}$ are Lagrangian multipliers, also known as "support values" that can either be positive or negative, and the corresponding data points, $x_{k}$, are the "support vectors". The support values are proportional to the errors at the data points. In the standard SVM case many of these support values are zero, but most of the least squares support values are non-zero. In [6] a conjugate gradient method is proposed for finding $b$ and $\alpha$, which are required for the SVM classifier in equation (1) .

\section{A. Multiclass Classification}

One-vs-one multiclass classification is based on binary LSSVMs. For $P$ distinct classes there are $\frac{P(P-1)}{2}$ hyperplanes that separate the classes with maximum margin. The hyperplanes with maximum margin are constructed in the LS-SVM training phase. The Decision Directed Acyclic Graph (DDAG) is a specific technique for one-vs-one multiclass classification [8]. The technique uses a tree structure to compare the test data to each of the hyperplanes. Through a series of elimination steps the best label is assigned to the input data. The LSSVM algorithm for DOA estimation is based on the DDAG architecture with each node containing a binary LS-SVM classifier of the $i^{t h}$ and $j^{t h}$ classes, refer to Figure 1.

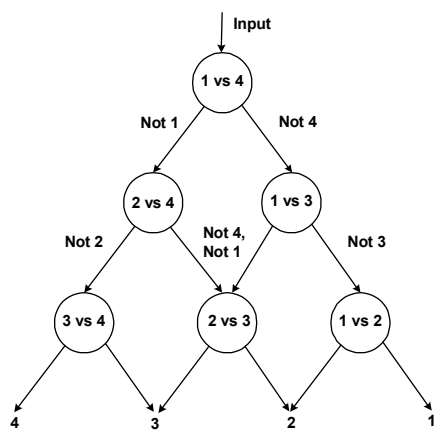

Fig. 1. Four Class DDAG

\section{SVMS AND DOA ESTIMATION}

The process of DOA estimation is to monitor the outputs of $D$ antenna elements and predict the angle of arrival of $L$ signals, $L<D$. The output vector for each incident signal from the antenna elements is $\overline{\mathbf{a}}\left(\theta_{l}\right)=$ $\left[\begin{array}{llll}1 & e^{-j k_{l}} & \ldots & e^{-j(D-1) k_{l}}\end{array}\right]^{T}$, and the vector of incident DOAs is $\boldsymbol{\theta}=\left[\theta_{1}, \ldots, \theta_{L}\right]$. With a training process, the learning algorithms generate DOA estimates based on the responses from the antenna elements, $\overline{\mathbf{a}}\left(\theta_{l}\right)$.

For the LS-SVM based approach to DOA estimation the output of the receiver is used to calculate the sample covariance matrix $\widehat{\mathbf{R}}_{r r}$ of the input data signal $\mathbf{x}_{r}(k), \widehat{\mathbf{R}}_{r r}=$ $\frac{1}{M} \sum_{k=K-M+1}^{K} \mathbf{x}_{r}(k) \mathbf{x}_{r}^{T}(k)$. The dimension of the observation matrix is $D \times M, M$ is ideal sample size (window length), and the dimension of the sample covariance matrix is $D \times D$. The principal eigenvectors, $\mathbf{v}_{1}, \ldots, \mathbf{v}_{D}$, are calculated via eigen decomposition (ED) or subspace tracking techniques. Each eigenvector is used to calculate a covariance matrix, $\widehat{\mathbf{R}}_{v v_{1}}, \ldots, \widehat{\mathbf{R}}_{v v_{D}}$.

The LS-SVM DOA estimation algorithm includes preprocessing, training, and testing steps.

\section{- Preprocessing for SVM Training}

1) Generate the $D \times N$ training vectors for the $P \mathrm{SVM}$ classes, $D$ is the number of antenna elements, $N$ is the number of input data samples. 2) Generate the $P$ sample covariance matrices, $\mathbb{C}$, with $M$ samples from the $D \times N$ data vector. 3) Calculate the signal eigenvector, $\mathbb{S}$, from each of the $P$ sample covariance matrices. 4) Calculate the $D \times 1$ projection vectors, $\mathbb{C} \cdot \mathbb{S}$, for each of the $P$ classes. 5) Store the projection vectors for the training phase and the eigenvectors for the testing phase.

- LS-SVM Training

1) With the $P$ projection vectors train the $\frac{P(P-1)}{2}$ nodes with the one-vs-one LS-SVM algorithm. 2) Store the LSSVM variables, $\alpha_{k}$ and $b$, refer to equation (1).

- Preprocessing for SVM Testing

1) Acquire $D \times N$ input signal from antenna array. 2) Generate the sample covariance matrix with $M$ samples from the $D \times N$ data vector. 3) Calculate the eigenvectors for the signal subspace and the noise subspace. 4) Generate the covariance matrices for each eigenvector

- LS-SVM Testing for the $i^{t h} / j^{\text {th }}$ DDAG Node.

1) Calculate two $D \times 1$ projection vectors with the desired eigenvector covariance matrix and the $i^{\text {th }}$ and $j^{\text {th }}$ eigenvectors from the training phase. 2) Test both projection vectors against the LS-SVM hyperplane for the $i^{t h} / j^{t h}$ node. 3) Calculate the mean value of the two LS-SVM output vectors (labels). Select the mean value that is closest to a decision boundary, 0 or 1 . Compare this value to the label definition at the DDAG node, then select the proper output label. 4) Repeat process for the next DDAG node in the evaluation path or declare the final DOA label.

- Error Control

1) Review the MSE calculations for the DDAG evaluation path. 2) Apply error control and validation measures to classify the label as either an accurate DOA estimate or as NOISE.

The algorithm presented in this section requires only the set of estimated eigenvectors, which are used to generate projection coefficients for the classification process. Table I includes three sets of projection vectors, each set corresponds 
TABLE I

PROJECTION COEFFICIENTS FOR MACHINE LEARNING BASED POWER CONTROL

\begin{tabular}{c||c|c|c}
\multicolumn{1}{c||}{} & \multicolumn{3}{c}{ Projection Coefficients } \\
& $25^{\circ}$ & $30^{\circ}$ & $40^{\circ}$ \\
\hline \hline 1 & $0.17+\mathrm{i} \cdot 0.86$ & $-0.20-\mathrm{i} \cdot 0.54$ & $0.00+\mathrm{i} \cdot 0.86$ \\
\hline 2 & $0.66+\mathrm{i} \cdot 0.05$ & $-0.82+\mathrm{i} \cdot 0.14$ & $0.73-\mathrm{i} \cdot 0.55$ \\
\hline 3 & $0.04-\mathrm{i} \cdot 0.73$ & $0.28+\mathrm{i} \cdot 0.96$ & $-1.01-\mathrm{i} \cdot 0.58$ \\
\hline 4 & $-1.08-\mathrm{i} \cdot 0.50$ & $1.04-\mathrm{i} \cdot 0.37$ & $0.06+\mathrm{i} \cdot 1.05$ \\
\hline 5 & $-0.60+\mathrm{i} \cdot 0.92$ & $-0.56-1 \cdot 1.01$ & $0.72-\mathrm{i} \cdot 0.61$ \\
\hline 6 & $0.60+\mathrm{i} \cdot 0.74$ & $-0.87+\mathrm{i} \cdot 0.64$ & $-0.92-\mathrm{i} \cdot 0.51$ \\
\hline 7 & $0.72-\mathrm{i} \cdot 0.56$ & $0.63+\mathrm{i} \cdot 0.62$ & $-0.03+\mathrm{i} \cdot 0.76$ \\
\hline 8 & $-0.52-\mathrm{i} \cdot 0.78$ & $0.51-\mathrm{i} \cdot 0.44$ & $0.45-\mathrm{i} \cdot 0.42$
\end{tabular}

to a different DOA. From a review of the data it is evident that the classes are not linearly separable. The data must be projected to a higher dimension feature space and tested against the separating hyperplane.

\section{A. Multilabel Capability for Multiple DOAs}

In DOA estimation for cellular systems, there can be multiple DOAs for a given signal. This results from multipath effects induced by the communication channel. The machine learning system must be able to discriminate between a small number of independent DOAs that include signal components with similar time delays. The machine learning algorithm then must generate multiclass labels, $y_{i} \in Y$, where $Y \in[-90,90]$ is a set of real numbers that represent an appropriate range of expected DOA values, and multiple labels $y_{i}, i=1 \ldots L$ for $L$ dominant signal paths. If antenna sectoring is used in the cellular system the multiclass labels are from the set $Y \in$ $\left[S_{i}\right]$, where $S_{i}$ is field of view for the $i^{t h}$ sector. The LS-SVM algorithm for DOA estimation assigns DOA labels to each eigenvector in the signal subspace. By repeating the DDAG cycle for each eigenvector the multiclass algorithm has the capability of assigning multiple labels to the input signal.

\section{B. Simulation Results}

Simulations of the LS-SVM DDAG DOA estimation algorithm are based on a complex system model that includes amplitude and phase distributions representative of the communication channel. The received signal at the receiver is modeled as

$x_{r}(t)=\sum_{d, l=1}^{D, L} \overline{\mathbf{a}}\left(\theta_{l}\right) \alpha_{d l} s_{d}\left(t-\tau_{d l}\right) \cos \left(w_{c}\left(t-\tau_{d l}\right)\right)+n_{d}(t)$.

This model includes $D$ antenna array elements with steering array vector $\overline{\mathbf{a}}\left(\theta_{l}\right)$ and additive Gaussian noise $n_{d}(t)$. The model assumes $L$ independent, resolvable signal paths. The multipath variable $\alpha_{d l}$ is defined as $\alpha_{d l}=$ $\rho_{d l} e^{j\left(2 \pi f_{c}\left(t-\tau_{d l}\right)+\phi_{c}\right)}$. The amplitude of the received signal $\rho_{d l}$, includes the transmitted power $\sqrt{p_{t}(t)}$ and the attenuation due to the link gain and shadowing $q^{l}$. This variable is modeled as a fixed, Rayleigh, Ricean, or log-normal distributed random variable. The Doppler shift for each resolvable path is defined by $f_{c}=\frac{v_{c} w_{c}}{2 \pi c} ; v_{c}$ is the velocity of the mobile in $\frac{m}{\mathrm{sec}}, w_{c}$ is the carrier frequency, and $c$ is the speed of propagation. A uniformly distributed carrier phase shift, $\phi_{c}$, and a time delay for each signal path, $\tau_{d l}$, are also included in the multipath variable, $\alpha_{d l}$. The CDMA spreading code, $s_{d}\left(t-\tau_{d l}\right)$, provides the processing gain at the correlator output.

The training and test signals are the complex outputs from the antenna array with eight elements. The basic multiclass LS-SVM system, presented in this paper, includes four DOA classes and six DDAG nodes. Figures 2 and 3 shows results for two four class DDAG architectures. The LS-SVM system in Figure 2 includes one degree per DOA class, Figure 3 shows ten degrees per DOA class. To completely test the LS-SVM DDAG's capabilities the simulations were automated to test a wide range of DOAs. The LS-SVM DDAG DOA estimation algorithm is extremely accurate. No misclassifications were logged. Additional simulations show that the LS-SVM DDAG system accurately classifies the DOAs for three to ten classes and DOA ranges from one degree to twenty degrees.

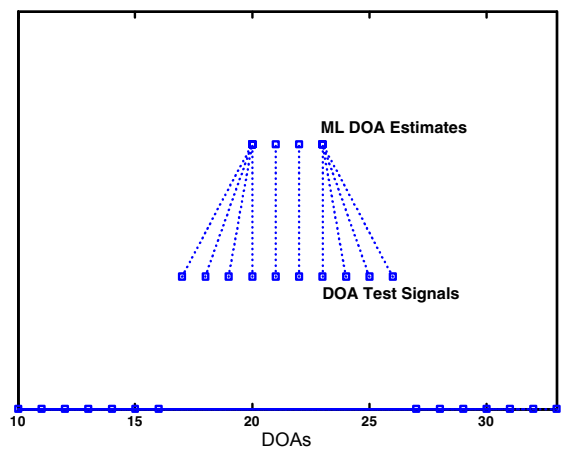

Fig. 2. LS-SVM for DOA estimation, four classes with one degree separation between each.

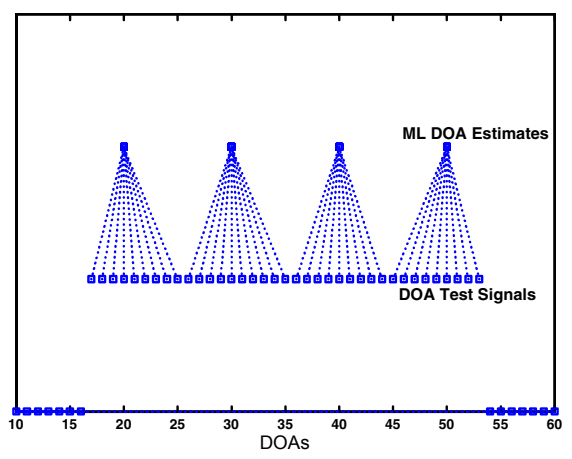

Fig. 3. LS-SVM for DOA estimation, four classes with ten degree separation between each.

\section{Classification Accuracy and Error Control}

For any estimation or classification algorithm the errors must be defined to evaluate the algorithm's performance and system capabilities. Specifically for classification algorithms, a high confidence level must exist along with a technique to automatically recognize misclassifications. Characterizing the 
performance of DDAG multiclass classification algorithms is a difficult task due to numerous possibilities with the evaluation path. In this section we present methods for performance characterization and error control.

\section{A. Decision Grids}

The decision grid (DG) technique was developed to track the DDAG evaluation path and generate statistics to characterize the confidence level of the DOA classifications. The empirical DG (E-DG) is a technique developed to quantify errors and add insight into the robustness of the LS-SVM DDAG architecture. E-DG's are automatically generated in the LS-SVM DDAG DOA estimation algorithm. The E-DGs tabulate the mean of the LS-SVM output label vectors at each DDAG node and level. The mean values are referred to as "decision statistics".

The unique design of this multiclass LS-SVM algorithm for DOA estimation includes testing the input data against two hyperplanes at the $i^{t h} / j^{t h}$ node. With this approach the two output vectors at each node are compared to one another. In a noise-free environment, with perfect classification, the two label vectors would be binary opposites, i.e. one output label would be a vector of $0^{\prime} s$ and the other output vector would be all $1^{\prime} s$. This technique enables computation of empirical mean square errors (MSEs), refer to Section IV-B.

Table II includes one standard DG and two E-DGs for a three class DDAG with a two degree DOA range per class. The two levels of a three class DDAG are equivalent to the first two levels of a four class DDAG, refer to Figure 1. The first DG shows the decision statistics for a noise free classification process, as presented by the deterministic evaluation paths. The first E-DG presents the decision statistics for a signal subspace eigenvector; the second E-DG presents the decision statistics for a noise subspace eigenvector. The output labels are the mean values of the vectors generated by the LS-SVM algorithm.

\section{B. Empirical MSEs}

The difficulty in tracking the performance of the LS-SVM DDAG DOA estimation algorithm is due to the numerous DDAG evaluation paths. For many DDAGs the evaluation paths can be determined based on the input data and the class definitions. How can decision statistics be applied to performance characterization?

The primary performance measure for the LS-SVM DDAG is the empirical MSE (E-MSE). The E-MSE is a measure of the empirical classification accuracy achieved at each DDAG node and is a technique that allows for real-time error tracking with only the decision statistics. The E-MSEs are calculated with the differences between the two LS-SVM decision statistics at each node in the evaluation path. For example, the E-MSE for a 3 class DDAG is calculated with only the EDG presented in Table II. The MSE for Class 2, Level 1 is $(|0.032-0.576|-1)^{2}=0.208$ and the MSE for Class 2, Level 2 is $(|1-0|-1)^{2}=0$.
TABLE II

THEORETIC DECISION GRID AND TEST CASES FOR SIGNAL AND NOISE EIGENVECTORS. THE DDAG SYSTEM INCLUDES 3 CLASSES WITH A 2 DEGREE DOA RANGE.

\begin{tabular}{c|c||c|c|c|c}
\multicolumn{1}{c||}{} & \multicolumn{2}{c|}{ Level 1 } & \multicolumn{2}{c}{ Level 2 } \\
\multicolumn{2}{c||}{ DOA } & Label 0 & Label 1 & Label 0 & Label 1 \\
\hline \hline \multicolumn{2}{c||}{ Decision Grid } & \multicolumn{4}{c}{} \\
\hline Class 1 & 1 & 0 & 1 & 0 & 1 \\
\hline & 2 & 0 & 1 & 0.5 & 0.5 \\
\hline Class 2 & 3 & 0.5 & 0.5 & $0 \backslash 1$ & $1 \backslash 0$ \\
\hline & 4 & 1 & 0 & 0.5 & 0.5 \\
\hline Class 3 & 5 & 1 & 0 & 1 & 0
\end{tabular}

\begin{tabular}{c|c||c|c|c|c}
\multicolumn{2}{c||}{ Signal Data } & \multicolumn{5}{c}{} \\
\hline Class 1 & 1 & 0 & 1 & 0 & 1 \\
\hline & 2 & 0 & 1 & 0.176 & 0.816 \\
\hline Class 2 & 3 & 0.032 & 0.576 & 1 & 0 \\
\hline & 4 & 0.952 & 0 & 0.808 & 0.496 \\
\hline Class 3 & 5 & 1 & 0 & 1 & 0
\end{tabular}

\begin{tabular}{c|c||c|c|c|c}
\multicolumn{2}{c||}{ Noise Data } & \multicolumn{5}{c}{} \\
\hline Class 1 & 1 & 0.328 & 0.752 & 0.232 & 0.896 \\
\hline & 2 & 0.376 & 0.744 & 0.256 & 0.904 \\
\hline Class 2 & 3 & 0.304 & 0.712 & 0.144 & 0.952 \\
\hline & 4 & 0.352 & 0.768 & 0.136 & 0.944 \\
\hline Class 3 & 5 & 0.384 & 0.776 & 0.184 & 0.944
\end{tabular}

\section{Error Control and Validation}

The design of the LS-SVM DDAG DOA estimation algorithm requires one step for a high confidence level in the classification process. For real-time applications the E-MSE provides two very important results:

1) A measure of confidence for the classification process.

2) A method for determining the dimension of the signal subspace.

Table III presents MSE data for a four class DDAG with $5^{\circ}, 10^{\circ}$ and $20^{\circ}$ DOA ranges. The MSE data is listed for each level in the DDAG's evaluation path, the average EMSE is also listed. The data shows that MSEs for a 4 class DDAG fluctuate little as a result of the DOA range. Likewise, additional simulations show that MSEs are relatively constant for different classes of DDAGs. LS-SVM DOA estimation data, not presented here, supports these trends for DDAGs architectures with three to ten classes and $1^{\circ}$ to $20^{\circ}$ DOA ranges per class.

The MSE data shows that there is a distinct and wide separation between signal and noise MSEs. The MSEs for signal subspace eigenvectors are less than $20 \%$, on average, of the MSEs for noise subspace eigenvectors. Based on the findings presented in this section the E-MSEs serve as reliable measure of confidence for the LS-SVM generated DOA labels. In addition, since the E-MSE is calculated with the LS-SVM DDAG output label vectors (with no prior information) realtime results provide required information on the confidence of the DOA estimation. With this E-MSE technique a LS-SVM DOA machine learning system automatically "decides" if a LS-SVM generated DOA label is accurate and assigned to a received signal, or inaccurate and a result of received noise. 
TABLE III

MSE PER DDAG LEVEL AND CLASS, FOUR CLASS DDAG WITH FIVE, TEN, AND TWENTY DEGREE DOA RANGES.

\begin{tabular}{|c|c|c|c|c|}
\hline & \multicolumn{3}{|c|}{ Level } & \multirow[b]{2}{*}{ Average } \\
\hline & 1 & 2 & 3 & \\
\hline \multicolumn{5}{|l|}{$5^{\circ}$ Range } \\
\hline E-MSE, Signal & 0.075 & 0.067 & 0.145 & 0.096 \\
\hline E-MSE, Noise & 0.843 & 0.636 & 0.460 & 0.646 \\
\hline \multicolumn{5}{|l|}{$10^{\circ}$ Range } \\
\hline E-MSE, Signal & 0.030 & 0.060 & 0.066 & 0.052 \\
\hline E-MSE, Noise & 0.718 & 0.743 & 0.654 & 0.705 \\
\hline \multicolumn{5}{|l|}{$20^{\circ}$ Range } \\
\hline E-MSE, Signal & 0.008 & 0.261 & 0.086 & 0.118 \\
\hline E-MSE, Noise & 0.545 & 0.552 & 0.646 & 0.581 \\
\hline
\end{tabular}

\section{Estimation of the Signal Subspace Dimension}

This paper presents a new application for multiclass classification. The research field of DOA estimation presents system level problems that can be solved with classification algorithms that include accurate error control and validation. For example, CDMA DOA estimation algorithms requires accurate knowledge of the signal subspace dimension, which provides a measure of received multipaths and is used in SIR estimation. Estimating the signal subspace dimension is not an easy task since the separation between the signal subspace and noise subspace eigenvalues may not be well defined. In addition, the errors associated with the sample covariance matrix add to the uncertainty. The information theoretic criteria [9] is an algorithm for determining the signal subspace dimension. This technique requires knowledge of all eigenvalues in the sample subspace and minimizing an MDL cost function. The LS-SVM DDAG algorithm presented in this paper is designed with an error control step. Since the algorithm classifies eigenvectors the signal subspace dimension is automatically calculated by monitoring the E-MSE and comparing it to values from other eigenvectors in the same data set.

Figure 4 displays the E-MSE for sixteen signals with DOAs spanning a four class DDAG. The DOA classes are defined at $30^{\circ}, 35^{\circ}, 40^{\circ}$, and $45^{\circ}$. The training and test vectors each consist of eighty data samples. MSE data is included for one signal in the signal subspace and one signal from the noise subspace. Each MSE data point was generated from 2000 independent data vectors containing signals incident upon an eight element antenna array. The received signals uniformly spans the DOA range of $30^{\circ}$ to $45^{\circ}$. There is a distinct separation between MSEs for signal eigenvectors and noise eigenvectors. The data presented in this paper proves that the MSE statistic is an accurate method for estimating the signal subspace dimension for all DOAs spanning the DDAG classes. Additional simulations show that the separation between the signal and noise subspaces is significant, and constant, for training vectors with 100 or more samples.

\section{CONClusion}

In this paper we presented a multiclass LS-SVM architecture for DOA estimation as applied to a CDMA cellular

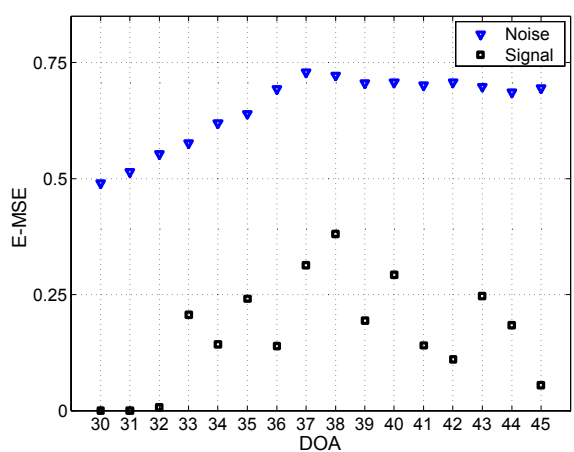

Fig. 4. MSE for DOAs spanning the four classes in the LS-SVM DDAG.

system. The LS-SVM algorithm for DOA estimation assigns DOA labels to each eigenvector in the signal subspace. By repeating the DDAG cycle for each eigenvector the multiclass algorithm has the capability of assigning multiple labels to the input signal. Simulation results show a high degree of accuracy, as related to the DOA classes and prove that the LSSVM DDAG system has a wide range of performance capabilities. The LS-SVM DOA estimation algorithm is superior to standard techniques due to the robust design that is insensitive to received SIR, Doppler shift, size of the antenna array, and the computational requirements are adaptable to the desired applications. The results show that the algorithm is accurate for a large range of DDAG performance independent of DDAG class or DOA range per class.

\section{REFERENCES}

[1] S. Chen, A.K. Samingan, and L. Hanzo, "Support Vector Machine Multiuser Receiver for DS-CDMA Signals in Multipath Channels", IEEE Transactions On Neural Networks, vol. 12, no. 3, pp. 604-611, May 2001.

[2] Daniel J. Sebald and James A. Bucklew, "Support Vector Machine Techniques for Nonlinear Equalization", IEEE Transactions On Signal Processing, vol. 48, no. 11, pp. 3217-3226, November 2000.

[3] Joseph C. Liberti, Jr. and Theodore S. Rappaport, Smart Antennas for Wireless Communications: IS-95 and Third Generation CDMA Applications, Prentice Hall, Upper Saddle River, NJ, 1999.

[4] Ahmed H. El Zooghby, Christos G. Christodoulou, and Michael Georgiopoulos, "A Neural Network-Based Smart Antenna For Multiple Source Tracking", IEEE Transactions On Antennas and Propagation, vol. 48, no. 5, pp. 768-776, May 2000

[5] Richard O. Duda, Perter E. Hart, and David G. Stork, Pattern Classification, Second Edition John Wiley \& Sons, New York, NY, 2001.

[6] J.A.K. Suykens, L. Lukas, P. Van Dooren, B. DeMoor, and J. Vandewalle, "Least Squares Support Vector Machine Classifiers: a Large Scale Algorithm", ECCTD'99 European Conf. on Circuit Theory and Design, pp. 839-842, August 1999.

[7] Johan A.K. Suykens, Tony Van Gestel, Jos De Brabanter, Bart De Moor, and Joos Vandewalle, Least Squares Support Vector Machines World Scientific, New Jersey, 2002.

[8] John C. Platt, Nello Christianini, and John Shawe-Taylor, "Large Margin DAGs for Multiclass Classification", in Advances in Neural Information Processing Systems, vol. 12, pp. 547-553, Cambridge, MA, MIT Press, 2000.

[9] Mati Wax and Thomas Kailath, "Detection of Signals by Information Theoretic Criteria", IEEE Transactions On Acoustics, Speech, and Signal Processing, vol. ASSP-33, no. 2, pp. 387-392, April 1985. 